\title{
NMR Study of Cluster-Assembled Nanophase Copper*
}

\author{
B. H. Suits ${ }^{1}$, M. Meng ${ }^{1}$, R. W. Siegel ${ }^{2}$, and Y. X. Liao ${ }^{2}$
}

1Michigan Technological University, Physics Department, Houghton, MI 49931

2Materials Science Division, Argonne National Laboratory, Argonne, IL 60439

To be published in Materials Research Society Symposium Proceedings Vol. 286 (Nanophase and Nanocomposite Materials), 1993; presented at the MRS 1992 FALL MEETING, November 30-December 4, 1992, Boston, MA.

*Work supported by the U. S. Department of Energy, BES-Materials Sciences, under Contract W-31-10 - Eng-38.

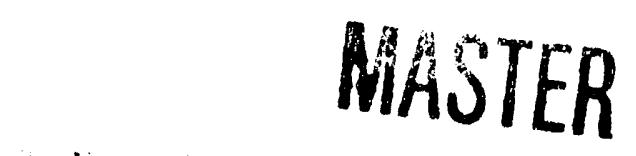




\title{
NMR Study of Cluster-Assembled Nanophase Copper
}

\author{
B. H. SUITS*, M. MENG*, R. W. SIEGEL**, and Y. X. LIAO** \\ * Michigan Technological University, Physics Department, Houghton, MI 49931. \\ * Materials Science Division, Argonne National Laboratory, Argonne, IL 60439.
}

\begin{abstract}
${ }^{63} \mathrm{Cu}$ and ${ }^{65} \mathrm{Cu}$ NMR spectra from cluster-assembled nanophase copper with an average grain size between 5 and $10 \mathrm{~nm}$ show a broadened peak, at the normal Knight-shifted frequency for copper metal, which arises from only the central $1 / 2$ to $-1 / 2$ transition. A very broad background is observed on either side of that peak. Some samples exhibit a second broad peak at a position normally associated with non-metallic copper. Pulsed NMR measurements of the central peak show that virtually all the copper signals are signif intly broadened and have a spin-spin relaxation time longer than larger grained copper samples. Line shape measurements, using spin echoes, as a function of delay between if excitation and measurement show there are a number of copper sites with longer relaxation times which have a significantly larger broadening. Those sites are tentatively identified as being at or near a grain boundary or free surface. A small orientation effect is observed indicating an anisotropy within the samples. An isochronal anneal of one sample showed significant, but not complete, line narrowing after an anneal at $450^{\circ} \mathrm{C}$ consistent with other nanophase metals which show grain growth above $40-50 \%$ of the melting temperature.
\end{abstract}

\section{INTRODUCTION}

Cluster assembled nanostructured materials, or "nanophase" materials, have interesting mechanical properties and both ceramics and metals have been studied using a variety of techniques. This paper reports nuclear magnetic resonance (NMR) measurements on cluster assembled copper samples. To the best of our knowledge, this is the first NMR study of nanophase materials. NMR probes the sample non-invasively and the NMR shifts and relaxation times provide information on the atomic and electronic structure surrounding an atom. Since nanophase materials contain a large number of atoms in grain boundaries and possibly at free surfaces, a local technique such as NMR can be hoped to be quite useful to characterize these materials.

\section{EXPERIMENTAL}

\section{Samples}

The samples were prepared using established techniques described elsewhere ${ }^{1,2}$. Copper (99.999\%) is evaporated in a slight helium atmosphere to form small clusters. Those clusters are collected then consolidated under uniaxial compression in vacuum to form a single piece of material in the form of a small disk approximately $1 \mathrm{~cm}$ in diameter and less than $1 \mathrm{~mm}$ in thickness. Grain size distributions are measured using dark field transmission electron microscopy (TEM) of a small portion of the sample. The samples look like normal copper metal to the eye.

The NMR measurements were performed on three different specimens with differing grain size distributions. Two of those samples were individual samples while the third NMR specimen consisted of six individual samples prepared under essentially identical 
conditions. For the combined samples, only two of the six were measured using the TEM. Since those two agreed so closely, it is assumed all six have approximately the same grain size distribution. The grain size distributions for the three NMR specimens are shown in figure 1 . Only one of the specimens which make up the combined sample is shown.

\section{NMR Measuremients}

Pulsed NMR measurements were performed using a home-built computer controlled NMR spectrometer using an $85 \mathrm{kG}$ magnetic field. Both naturally occurring isotopes of Copper, ${ }^{63} \mathrm{Cu}$ and ${ }^{65} \mathrm{Cu}$, were used. Due to the large line widths and interference from other common elements $\left({ }^{23} \mathrm{Na},{ }^{27} \mathrm{Al},{ }^{13} \mathrm{C}\right)$ for ${ }^{63} \mathrm{Cu}$, most measurements, and all those presented here, where made using the slightly less sensitive ${ }^{65} \mathrm{Cu}$ resonance which occurs just above $100 \mathrm{MHz}$ in our field. For these measurements, though the bandwidth of the receiver is quite large, the bandwidth limitations due to the finite size of the $\mathrm{rf}$ excitation are about $\pm 3 \mathrm{ckHz}$. Very wide line spectra were collected by plotting the area under an echo signal as a function of spectrometer frequency.

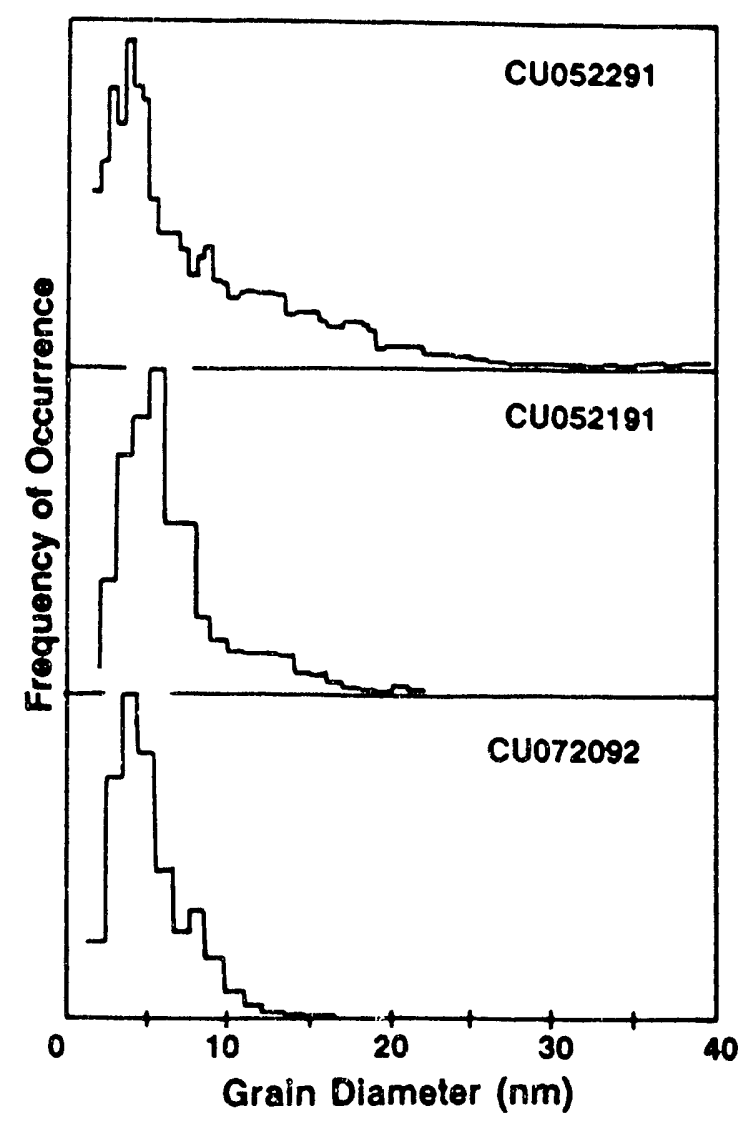

Fig. 1 - The grain size distributions measured using dark-field electron microscopy for three copper samples. NMR measurements of the upper two were made as individual samples, the bottom sample was one of six made under similar conditions which were stacked and measured together.

Both the "solid echo" and Hahn echo were used in addition to some single pulse, free induction decay (FID) measurements. The solid echo consists of two pulses of equal duration which are $90^{\circ}$ out of phase and separated by a time 9 and is useful for refocusing the effects of the nuclear-nuclear, or dipolar coupling. The Hahn echo also uses two pulses but the second is twice the length of the first. The Hahn echo refocuses inhomogeneous broadening effects--when different sites respond at different frequencies-but not the dipolar interaction. For most measurements, the lengths of the pulses were chosen to maximize the signal. The NMR response is spread out over a very wide frequency range leading to weak signals requiring extensive signal averaging. The single samples are relatively small and each spectra was acquired over a period of just over a week. For the combined sample, a spectrum $1 \mathrm{MHz}$ wide can be obtained in a few hours.

The central line of the nanophase copper and several off-the-shelf copper samples (wire, foil, and lathe shavings), presumed to be large grained, were measured using Fourier transform NMR techniques. Only a very small Hahn echo could be observed at short times for the off-the-shelf copper.

\section{RESULTS AND DISCUSSION}

\section{Spectra}

In addition to the Zeeman interaction which provides the majority of the splitting 
between the nuclear ground state ener $e_{k}$ levels, there are three important interactions to consider for these $\mathrm{Cu}$ materials, the $\mathrm{K}_{\mathrm{r}}$, $t$ shift, the effects of electric field gradients (efg), and the nuclear spin-spin interaction. The Knight shift and the electric field gradients cause shifts in the frequency of the NMR response while the spin-spin interactions influence the width and the spin-spin relaxation time but provide no net shift.

The nuclear magnetic resonance (NMR) response to a radio-frequency perturbation from a nucleus in a metal will cccur at a slightly different (usually higher) frequency than the response from the same nucleus in a non-metallic environment due to the Knight shift. The Knight shift is a result of the interaction between the nucleus and the conduction electrons. The NMR frequency for a nucleus with a gyromagnetic ratio $\gamma$ which is in a magnetic field $\mathrm{H}_{\mathrm{o}}$ can be written as:

$$
v=\gamma H_{o}(1+K)
$$

The Knight shift, $\mathrm{K}$, for copper metal, referenced to $\mathrm{CuCl}$, is ${ }^{3} 0.232 \%$.

If the nucleus used for study has a nuclear spin $I>1 / 2$ then one can expect electric field gradients in the sample to influence the NMR spectrum if the environment has a symmetry lower than cubic. To first order this will result in a shift of the NMR frequency of

$$
v_{m, m-1}=v_{q}(2 m-1)\left(3 \cos ^{2} \theta-1-\eta \cos 2 \phi \sin ^{2} \theta\right) / 4
$$

for the transition from level $m$ to level $m-1(m=I, I-1, \ldots-I+1)$. There is no first order shift for the $1 / 2$ to $-1 / 2$ transition (if present) and the other transitions will occur in pairs equally spaced about the center position. The parameters $v_{\mathrm{q}}$ and $\eta$ characterize the strength and symmetry of the electric field gradient respectively and the angles refer to Euler angles from the applied magnetic field direction. Expressions for the second order shift can be found in ref. 4 .

For ${ }^{63} \mathrm{Cu}$ and ${ }^{65} \mathrm{Cu}$, both of which have a spin $I=3 / 2$, there should be no electric field gradients in crystalline copper metal due to the cubic symmetry surrounding a copper site. The presence of strain, grain boundaries, or of other defects which destroy the cubic symmetry in the neighborhood of an observed nucleus will result in electric field gradients which can be characterized using NMR spectra. For nanophase $\mathrm{Cu}$ one would expect to find a distribution of values for $v$ and $\eta$ and all possible orientations as well.

The spin-spin interaction, or dipolar coupling, between nuclei is due to the coupling between adjacent nuclear magnetic moments. The direct dipolar coupling in solid samples results in a line broadening which depends strongly on the distance between the nuclei. Indirect couplings between nuclei through the electrons surrounding the atoms may also exist and also depend on the distance between nuclei. Previous measurements on copper indicate

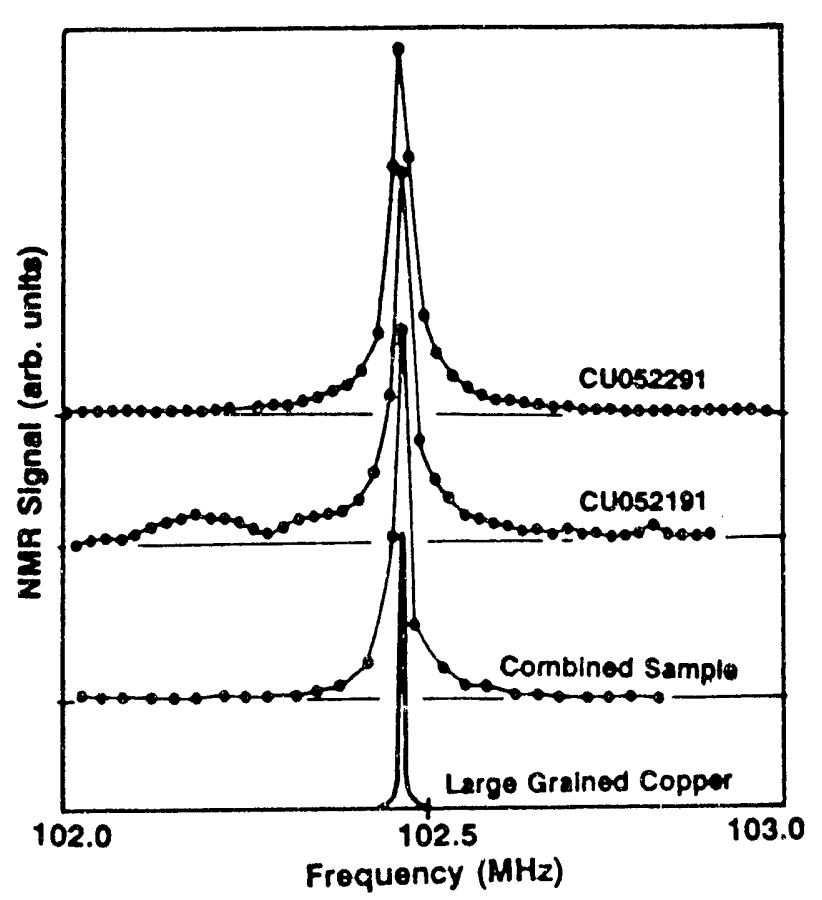

Fig. 2 - NMR spectra of three nanophase Cu samples measured by plotting the area under a NMR echo as a function of excitation frequency. All show a strong peak at the Knight shifted position for normal copper (bottom trace). 
the line width can be well described with only the direct interactions ${ }^{5}$.

Spectra obtained by plotting the area under spin echoes as a function of frequency for the three samples are shown in figure 2 . They all have a relatively sharp peak near the position where the Knight shifted signal from copper metal is normally observed and a weaker broad background spread out well over $1 \mathrm{MHz}$. Measurements of both ${ }^{63} \mathrm{Cu}$ and ${ }^{65} \mathrm{Cu}$ were made and little difference could be seen between the two.

One sample (CU052191) showed a second broad peak at a frequency normally associated with copper in non-metals. The conditions under which this peak is observed are under study, however measurements of $\mathrm{CuO}$ and $\mathrm{Cu}_{2} \mathrm{O}$ clearly show that it is not due to either of these common oxides.

\section{Transient Response}

One of the difficulties interpreting the Cu signals is to determine whether the signal at the Knight shifted position is due to $\mathrm{Cu}$ in a cubic environment or is it simply due to the relatively unperturbed $1 / 2$ to $-1 / 2$ transitions of nuclei which are not in a cubic environment. The tivo extremes can be easily distinguished by measuring the strength of the NMR response as a function of applied pulse length. If there is a sufficiently large efg present to move the $\pm 3 / 2$ to $\pm 1 / 2$ transitions outside the $\mathrm{rf}$ excitation bandwidth, the response of the $1 / 2$ to $-1 / 2$ transition acts like an "effective spin-1/2" in a magnetic field but with a scale factor on the gyromagnetic ratio used for the coupling to the rf field. In the case of copper, the coupling is a factor of 2 larger than for copper in a cubic environment.

The central peak of the nanophase samples were compared to large grained copper metal samples, and a factor of $2( \pm 10 \%)$ was observed. A sample which has a mix of cubic and non-cubic environments would be expected to show some intensity with the factor of 2 increase, and some without. No measurable intensity was seen which did not have this factor of 2 increase indicating that the vast majority of the copper sites are noncubic (that is, they are distorted from cubic). This behavior has been observed to some degree in other copper samples which contain a large number of mechanically produced defects ${ }^{s}$.

\section{Spin-Spin Reiaxation}

To investigate the nature of the broadening of the central peak relative to large grained copper samples, the spin-spin relaxation time, $\mathrm{T}_{2}$, was measured using the amplitude of Hahn echoes as a function of pulse spacing. The amplitudes measured over the first decade of the decay ivllow a simple exponential decay, $\exp \left(-2 \tau / \mathrm{T}_{2}\right)$, with $\tau$ the time between $\mathrm{rf}$ pulses and a value of $\mathrm{T}_{2}=(96 \pm 10 \mathrm{k} u \mathrm{~s}$. This corresponds to a Lorentzian line width with a full width at half the maximum height (FWHM) of $3.5 \mathrm{kHz}$. The FWHM of the spectra, which appear Gaussian, for the large grained copper samples is $8.7 \mathrm{kHz}$. While some narrowing is expected in the presence of quadrupo!e splitting ${ }^{6}$, the size of the difference observed here is well out of the range expected (no more than $80 \%$ ) for the direct dipole broadening if all else is kept constant. In any event, the broadening due to the spin-spin interaction is significantly less for the nanophase materials compared to larger grained samples and does not explain the broader lines observed in the nanophase materials.

Since the spin-spin relaxation time depends on the spacing and the number of the neighbors near a nucleus and one can expect the atomic spacings to differ, for example, at or near a grain boundary, spectra were irivestigated as a function of the Hahn echo delay time, $\tau$, to look for changes associated with a distribution of relaxation times. 
Indeed, the spectral line shapes change significantly for longer times. Figure 3 shows a series of Fourier transform spectra taken with different delay times. Spectra in figure 3 are scaled to have the same height. Note that the spectra are somewhat bandwidth limited and hence the bruadening effect may be even bigger than what appears in figure 3 . As the delay increases, the spectra become much broader and more asymmetric towards lower frequency. A measurement for even longer times ( $r$ $=400 \mathrm{~s}$ ) gave a very weak response, but with an echo width comparable to the pulse lengths used indicating a line width larger than the excitation bandwidth.

The components of the spectrum which are more visible at long times are those with weaker spin-spin interactions. Without getting into the details of the spin-spin interaction, one can say that in general, the less dense the material, the weaker the interaction. Since the average density is expected to be reduced in the vicinity of a grain boundary or a free surface, and the disorder greater (leading to a larger line width) we tentatively identify these signals with nuclei at or near a grain boundary and/or a free surface. Further work will be necessary to separate out the spectra with shorter and longer relaxation times.

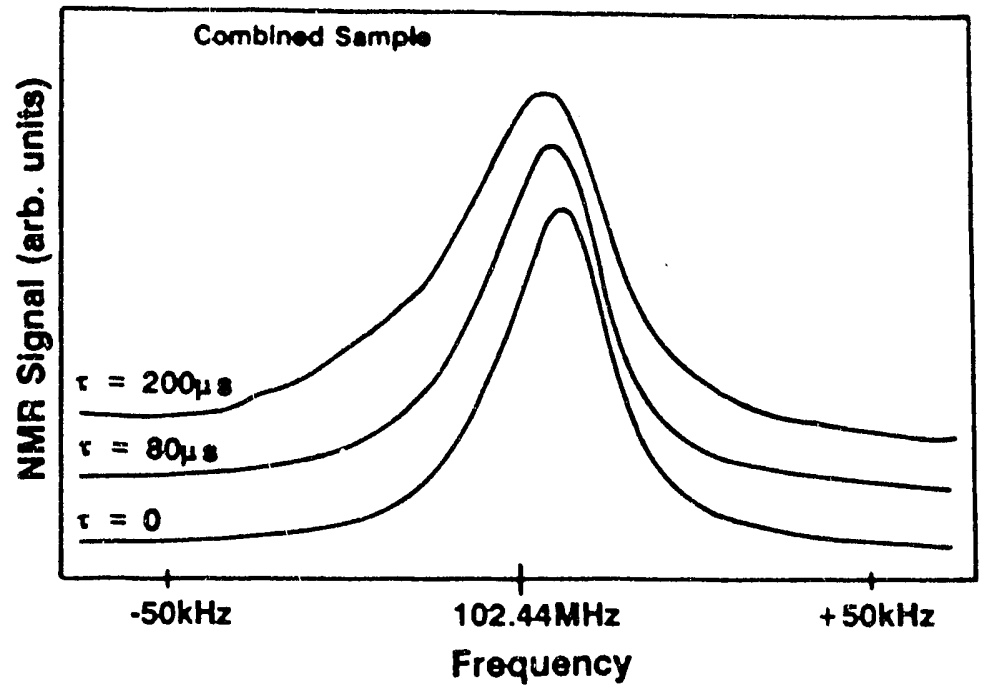

Fig. 3 - NMR fourier transform spectra obtained for different delay times following off excitation. The trace labelled $\tau=0$ is obtained using a single pulse free induction decay following a receiver dead time of $15 \mu \mathrm{s}$, the other two are obtained from Hahn spin echoes and $\tau$ refers to the time between the two pulses. For the Hahn echoes, the total time from the first excitation to the signal is $2 \tau$.

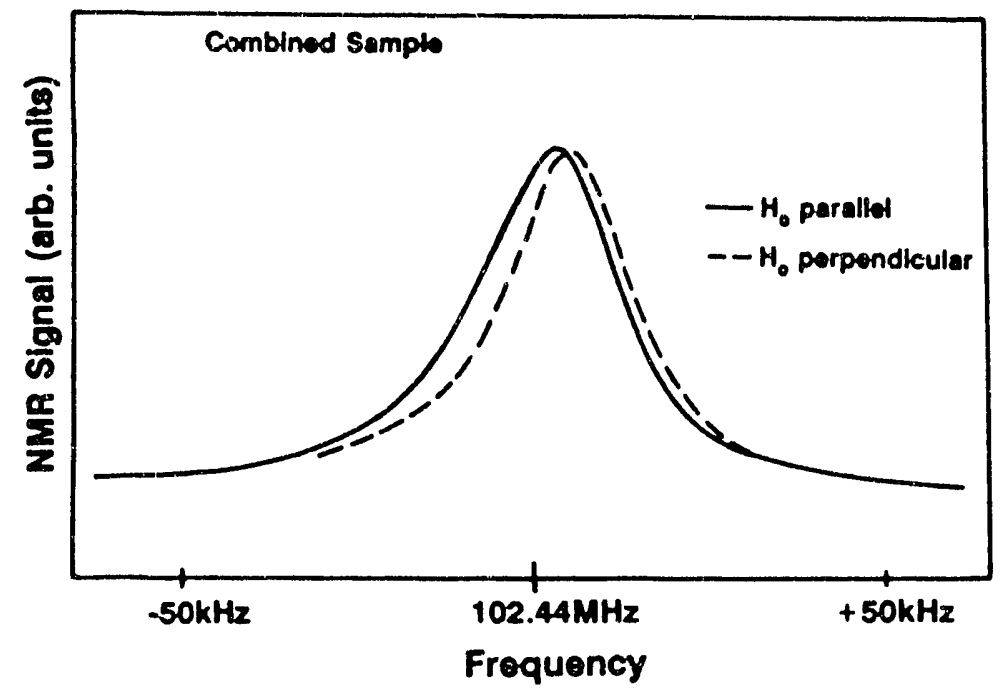

Fig. 4 - NMR fourier transform spectra from Hahn echoes obtained at longer delay times $(\tau=200 \mu \mathrm{s})$ as a function of orientation between the applied magnetic field and the compression axis used during sample preparation. Measurements at short times and for large grained copper samples do not show this orientation effect.

\section{Orientation Effects}

The broadening on the spectra discussed in the previous section was observed to have a significant orientation dependence as the sample was rotated in the magnetic field as shown in figure 4. Spectra measured with very short time delays and spectra measured on a sample of approximately the same size made from copper foil did not show this effect. The samples were positioned by eye. The fact that short time measurements do 
not show this effect rules out the possibility that it is due to the bulk magnetic susceptibility and sample geometry. Hence there is a part of the sample which is preferentially oriented. A measurement at an intermediate angle showed an effect between the extremes shown in figure 4 . We note that normal copper is slightly diamagnetic and hence the presence of voids would tend to cause a broadening toward higher frequency, rather than lower ${ }^{7}$ The magnetic susceptibility of nanophase $\mathrm{Cu}$ has not been measured.

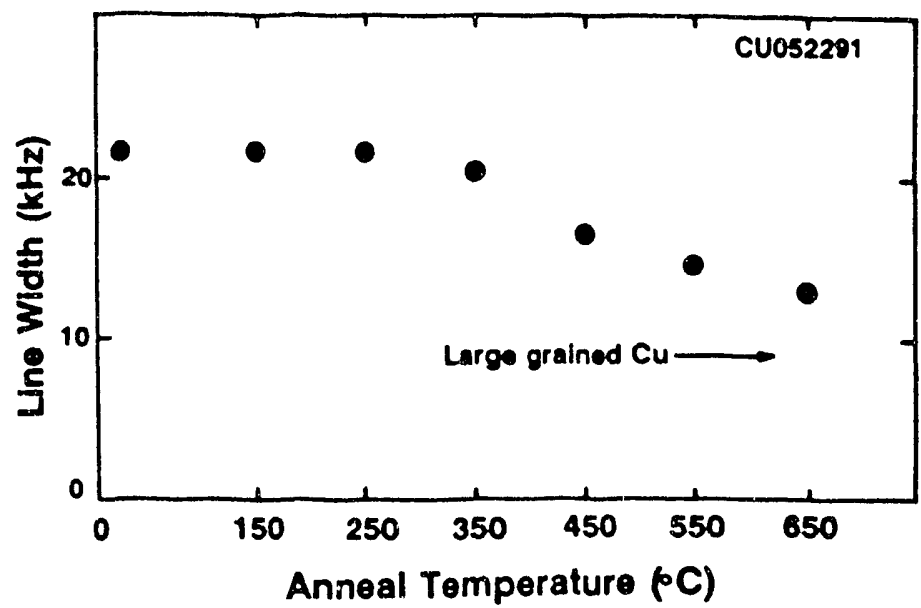

Fig. 5 - The line width (FWHM) of room temperature fourier transform measurements as a function of annealing temperature. Anneals were for 30 minutes each.

\section{Isochronal Anneal}

Room temperature NMR spectra of one of the samples were made following 30 minute anneals in an argon atmosphere at increasingly elevated temperatures. The FWHM of spectra obtained by fourier transformation of echo signals as a function of anneal temperature are shown in figure 5. The line width remains constant up to $350^{\circ} \mathrm{C}(620 \mathrm{~K})$, then narrows significantly, though not completely at higher temperatures. This behavior is consistent with other nanophase materials where significant grain growth occurs at 40 $50 \%$ of the melting temperature ${ }^{8}$ (1356K for copper). These measurements were made with the compression axis perpendicular to the applied magnetic field and a solid echo pulse sequence with a moderate delay time.

Work at Argonne National Laboratory supported by U.S. Dept. of Energy, BES-DMS, under Contract W-31-109-Eng-38.

\section{References}

1. G. W. Nieman, J. R. Weertman, and R. W. Siegel, J. Mater. Res. 6, 1012 (1991).

2. R. W. Siegel, MRS Bulletin, October 1990, pg 60.

3. W. D. Knight, Solid State Physics 2, 93 (1956).

4. G. H. Stauss, J. Chem. Phys. 40, 1988 (1964).

5. O. Kanert, Physica Status Solidi 32, 667 (1969).

6. A. Abragam, Principles of Nuclear Magnetism, Clarendon Press, Oxford (1961).

7. D. C. Ailion, T. A. Case, D. D. Blatter, A. H. Morris, A. G. Cutillo, C. H. Durney, and S. A. Johnson, Bulletin of Magnetic Resonance 6 No. 3, 131 (1984).

8. R. W. Siegel, Annual Review of Materials Science 21, 559 (1991). 

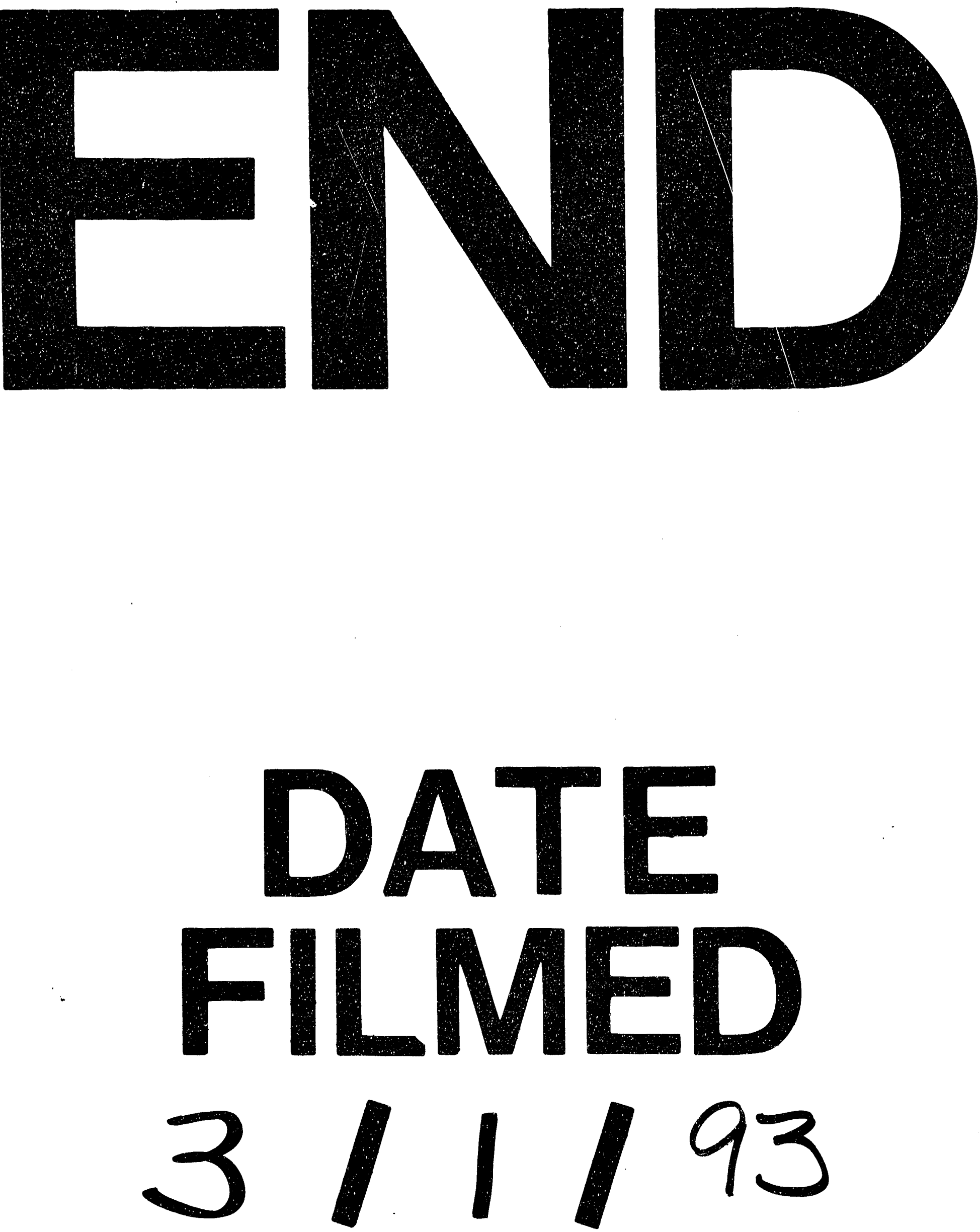
